\title{
Segurança e Eficácia Tardia do Stent com Eluição de Paclitaxel no Infarto Agudo do Miocárdio: um Estudo Caso-Controle
}

\author{
Fernando de Martino1, 2, João Luiz de Alencar Araripe Falcão', Expedito Eustáquio Ribeiro', \\ Pedro Alves Lemos Neto ${ }^{1}$, André Gasparini Spadaro', Gilberto Guilherme Ajjar Marchiori', \\ Carlos A. H. Campos ${ }^{1}$, Marco Antônio Perinn ${ }^{1}$, Pedro Eduardo Horta ${ }^{1}$, Breno de Alencar Araripe Falcão ${ }^{1}$, \\ Luiz Junya Kajita' ${ }^{1}$, Rone Padilha², Sérgio Prata², José Abud², Eulógio Emílio Martinez Filho
}

\section{RESUMO}

Introdução: A segurança e a eficácia tardia de stents com eluição de paclitaxel (SEP) ainda são debatidas no infarto agudo do miocárdio (IAM). Método: Para avaliar o desempenho dos SEP em pacientes com IAM tratados com intervenção coronária percutânea (ICP), 60 pacientes foram selecionados para uma análise tipo caso-controle, comparando um grupo com SEP (Taxus ${ }^{\mathrm{TM}}$ ) e um grupo controle com o stent convencional análogo (Express IITM). Os parâmetros para escolha dos controles foram o comprimento e o diâmetro do stent, e a presença de diabetes melito. Eventos cardíacos adversos maiores foram avaliados durante o seguimento clínico. Reestudo angiográfico tardio foi realizado em todos os pacientes. Avaliação ultra-sonográfica foi realizada no grupo SEP. Resultados: As características basais foram semelhantes entre os grupos. Após 24,9 $\pm 5,3$ meses de acompanhamento, houve uma revascularização da lesão-alvo (RLA) no grupo SEP (nova ICP), e uma morte não-cardíaca, um reinfarto e seis RLA (duas cirurgias e quatro novas ICP) no grupo controle. O grupo SEP apresentou menor risco de nova revascularização (3,3\% vs. 23,3\%; $p=0,02)$. Ao reestudo angiográfico realizado, em média, $8,7 \pm 3,1$ meses após o procedimento índice, a perda angiográfica tardia foi de $0,21 \pm 0,29 \mathrm{~mm}$ vs. $0,60 \pm 0,76$ $\mathrm{mm}(\mathrm{p}=0,01)$ e a taxa de reestenose binária foi de $3,3 \%$ vs. $33,3 \%(p=0,002)$ nos grupos SEP e controle, respectivamente. À ultra-sonografia, o grupo SEP apresentou obstrução neointimal intra-stent porcentual média de 4,7 \pm 6,8\%. Má aposição do stent foi observada em 8 (34,8\%)

\section{SUMMARY}

Safety and Long-Term Efficacy of PaclitaxelEluting Stent for Acute Myocardial Infarction: A Case-Control Study

Background: The long-term safety and efficacy of paclitaxeleluting stents (PES) remains debated for patients with acute myocardial infarction (AMI). Method: To evaluate the performance of PES in patients with $\mathrm{AMI}$ treated with percutaneous coronary intervention $(\mathrm{PCI}), 60$ patients were subjected to a matched analysis comparing PES stent (Taxus ${ }^{T M}$ ) with an analogous bare metal stent (Express $I^{T M}$ ) control group. Matching parameters included stent length and diameter, and diabetes mellitus. Major adverse cardiac events were evaluated during follow up. Late angiographic restudy was obtained for all patients. Intravascular ultrasound (IVUS) evaluation was performed in PES group. Results: Baseline and procedural characteristics were similar between PES and control groups. After a mean follow-up of $24.9 \pm$ 5.3 months, there was 1 target lesion revascularization (TLR) in the PES group (re-stenting) and 1 non cardiac death, 1 re-AMI and 6 TLR (2 surgical revascularizations and 4 re- $\mathrm{PCl}$ ) in the control group. Patients of PES group had a lower risk of repeat revascularization (3.3 vs. 23.3\%; $\mathrm{p}=0.02)$. At angiographic restudy $8.7 \pm 3.1$ months after index procedure, angiographic late loss was $0.21 \pm 0.29$ vs. $0.60 \pm 0.76 \mathrm{~mm}(\mathrm{p}=0.01)$ and the binary reestenosis rate was 3.3 vs. $33.3 \%(p=0.002)$ in the PES vs. control groups respectively. At IVUS evaluation, average percent

\footnotetext{
1 Serviço de Hemodinâmica do Instituto do Coração do Hospital das Clínicas da Faculdade de Medicina da Universidade de São Paulo - São Paulo, SP.

2 Instituto Uberabense de Cardiologia Invasiva - Uberaba, MG. Correspondência: João Luiz de Alencar Araripe Falcão. Av. Dr. Enéas Carvalho de Aguiar, 44 - 3o andar (Serviço de Hemodinâmica) - Cerqueira César - São Paulo, SP - Brasil - CEP 05403-000

Recebido em: 22/8/2007 - Aceito em: 12/10/2007
} 
Martino F de, et al. Segurança e Eficácia Tardia do Stent com Eluição de Paclitaxel no Infarto Agudo do Miocárdio: um Estudo CasoControle. Rev Bras Cardiol Invas 2007; 15(4): 343-349.

pacientes. Conclusão: O SEP (Taxus ${ }^{\mathrm{TM}}$ ) mostrou-se seguro e efetivo em pacientes com IAM, durante seguimento clínico de dois anos.

DESCRITORES: Infarto do miocárdio. Angioplastia transluminal percutânea coronária. Stents. Paclitaxel. Segurança. Reestenose coronária.

0 s stents com eluição de paclitaxel (SEP) têm se mostrado superiores aos stents metálicos convencionais, em pacientes tratados com intervenção coronária percutânea (ICP) eletiva ${ }^{1,2}$. Entretanto, a adição de uma potente droga antiproliferativa ao stent e seu conseqüente impacto sobre a resposta cicatricial vascular têm gerado preocupações com relação à implantação de stents com eluição de drogas em pacientes com mais alto risco para complicações trombóticas. De fato, o desempenho clínico dos SEP ainda é debatido em pacientes com síndrome coronária aguda.

Uma subanálise com seguimento de um ano do estudo TAXUS-IV demonstrou que pacientes com síndrome coronária aguda que receberam SEP (Taxus ${ }^{\mathrm{TM}}$ ) tiveram taxa significativamente menor $(3,9 \%$ vs. $16,0 \%$; $p<0,0001)$ de revascularização da lesão-alvo, comparativamente ao grupo controle que recebeu stent metálico convencional (Express ${ }^{\mathrm{TM}}$ ), com baixa taxa de trombose de stent $(0,8 \%)^{3}$. Entretanto, pacientes com infarto agudo do miocárdio (IAM) com supradesnivelamento do segmento ST foram excluídos desse estudo. No estudo Paclitaxel Eluting Stent Versus Conventional Stent in ST-Segment Elevation Myocardial Infarction (PASSION), maior estudo randomizado nesse tema, que avaliou o uso de SEP em pacientes com IAM com supradesnivelamento do segmento ST tratados com ICP primária, houve apenas tendência não-significativa a menor taxa de eventos cardíacos adversos maiores (ECAM) em pacientes tratados com SEP em relação ao grupo tratado com stent convencional (não revestido), após um ano de seguimento clínico $(8,8 \%$ vs. 12,8\%; risco relativo ajustado de 0,63 ; intervalo de confiança de $95 \%$ entre 0,37 e 1,$07 ; p=0,09)^{4}$. Em outro estudo, uma série consecutiva de pacientes com IAM tratados com SEP foi comparada a uma coorte de pacientes que recebeu stents com eluição de sirolimo nos meses precedentes ${ }^{5}$. Os pacientes do grupo SEP foram mais freqüentemente submetidos a novos procedimentos de revascularização após 12 meses, principalmente em decorrência de maior taxa de trombose de stent durante o acompanhamento clínico.

Neste estudo, procurou-se avaliar a evolução tanto clínica como angiográfica de pacientes com IAM tratados com SEP (Taxus ${ }^{\mathrm{TM}}$ ) comparados a um grupo controle que recebeu stent convencional análogo, com a mesma plataforma metálica não revestida (Express II ${ }^{\mathrm{TM}}$ ). in-stent neointimal obstruction of PES group was $4.7 \pm$ $6.8 \%$. Stent malapposition was observed in 8 patients (34.8\%). Conclusion: Paclitaxel-eluting stents (Taxus ${ }^{\top M}$ ) appeared safe and effective in patients with acute myocardial infarction at a two-year period follow-up.

DESCRIPTORS: Myocardial infarction. Angioplasty, transluminal, percutaneous coronary. Contenedores. Paclitaxel. Safety. Coronary restenosis.

\section{MÉTODO}

\section{População do estudo e protocolo}

Entre março de 2004 e março de 2005, 182 pacientes com IAM foram tratados com ICP, no Instituto do Coração do Hospital das Clínicas da Faculdade de Medicina da Universidade de São Paulo (InCor/HCFMUSP). O IAM foi diagnosticado como um episódio de precordialgia prolongado, não aliviado com o repouso ou com o uso de nitratos, e associado à elevação de marcadores cardíacos (creatina quinase fração $\mathrm{MB}[\mathrm{CKMB}]>2$ vezes o limite superior da normalidade). Pacientes com ou sem elevação do segmento ST foram elegíveis para este estudo. Para admissão no estudo, as lesões deveriam ser tratadas com um único stent. Classificação em Killip III ou IV (choque cardiogênico) e incapacidade de cruzar a lesão com a cordaguia foram critérios de exclusão. Após assinatura do termo de consentimento informado, 30 pacientes foram tratados com implante de SEP (Taxus ${ }^{\mathrm{TM}}$, Boston Scientific, Estados Unidos). Um grupo controle de 30 pacientes foi selecionado entre os casos previamente tratados com stent convencional similar (Express II $^{\mathrm{TM}}$, Boston Scientific, Estados Unidos). A escolha dos pacientes do grupo controle baseou-se em quatro parâmetros: quadro clínico à admissão, diabetes, comprimento do stent, e diâmetro do stent (Figura 1). Quando mais de um paciente com critérios de pareamento adequados fosse identificado, apenas um era selecionado

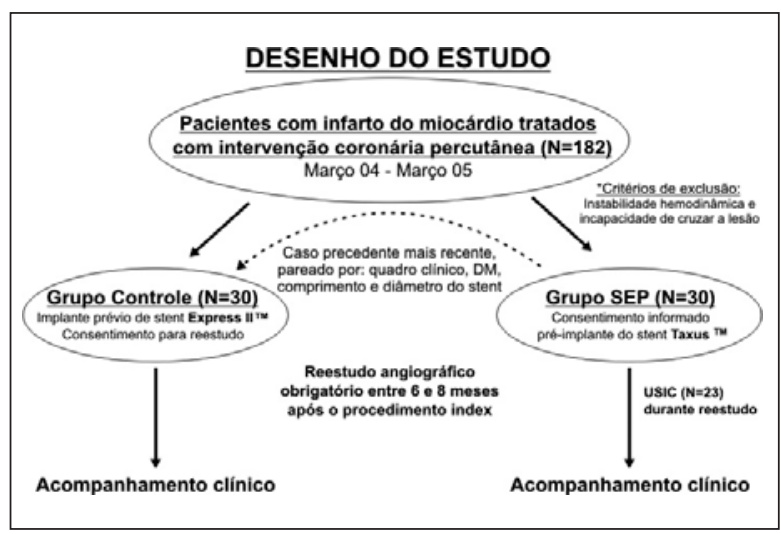

Figura 1 - Fluxograma do estudo. 
aleatoriamente para compor o grupo controle. Se o pareamento perfeito para diâmetro e comprimento do stent empregado não fosse encontrado, o paciente com as características do stent mais próximas ao empregado era escolhido para o grupo controle. O pareamento de cada caso foi realizado logo após a ICP e foi cego quanto ao desfecho clínico do paciente selecionado como controle. Após a seleção "cega", os pacientes do grupo controle foram contatados e convidados a participar do estudo, sendo incluídos os pacientes que concordaram em realizar reestudo angiográfico (consentimento informado). O protocolo de estudo foi revisado e aprovado pelo comitê de ética local.

Os pacientes foram tratados seguindo as técnicas rotineiras do serviço, e o uso de medicações adjuntas foi deixado a critério do operador. Após o cruzamento bem-sucedido da lesão com a corda-guia, o implante do stent foi realizado com ou sem pré-dilatação com balão. Aspirina foi prescrita como uso contínuo para todos os pacientes. Clopidogrel foi prescrito por um mês para o grupo controle, e por seis meses para o grupo SEP.

\section{Definições de eventos cardíacos adversos maiores}

Os pacientes foram seguidos, prospectivamente, para avaliar a ocorrência de ECAM: 1. morte, 2. reinfarto ou 3. revascularização da lesão-alvo. Reinfarto foi diagnosticado pela ocorrência de novos sintomas e/ou alterações eletrocardiográficas, associados com nova elevação de CKMB nas primeiras 48 horas após ICP (valor aferido pelo menos 1,5 vez maior que o anteriormente dosado), ou elevação de CKMB acima de 3 vezes o limite superior normal, se após 4 horas do infarto índice ${ }^{6,7}$. Revascularização da lesão-alvo foi definida pela realização de qualquer procedimento de revascularização (cirúrgico ou percutâneo) dirigido à lesão localizada no segmento arterial que recebeu stent, sendo aí incluídos, além do stent, os $5 \mathrm{~mm}$ proximais e distais às bordas do stent.

\section{Definições de trombose de stent}

Durante o seguimento clínico dos pacientes, procurou-se também avaliar a ocorrência de trombose do stent. Neste estudo, considerou-se o diagnóstico de trombose definitiva apenas quando o paciente apresentou quadro clínico de síndrome coronária aguda, acompanhado por achado angiográfico compatível com trombo ou oclusão do vaso no sítio de implante do stent. O achado anatomopatológico de trombo intra-stent também confirmaria o diagnóstico de trombose de stent. O diagnóstico de provável trombose de stent foi definido se houvesse óbito inexplicado nos primeiros 30 dias pós-ICP ou IAM relacionado ao vaso tratado, mesmo sem confirmação angiográfica, se posteriormente não fosse identificada outra lesão culpada. Atribuiu-se a uma possível trombose de stent qualquer óbito inexplicado ocorrido após 30 dias do implante do stent.

\section{Análise angiográfica coronária quantitativa ( $A C Q)$}

Após a administração de nitroglicerina intracoronária, a aquisição de imagens angiográficas padrão para avaliação de estenose coronária foi realizada utilizando pelo menos duas projeções angiográficas, as quais eram repetidas ao final do procedimento (apenas para o grupo SEP) e no momento do reestudo angiográfico. Os cineangiogramas foram analisados por um observador cego quanto ao stent empregado. As características morfológicas qualitativas foram avaliadas utilizando critérios padronizados. O diâmetro luminal mínimo da lesão e os diâmetros dos segmentos de referência foram medidos antes e após o implante do stent e ao reestudo angiográfico. O reestudo angiográfico foi realizado em todos os pacientes do estudo entre seis e oito meses após o procedimento índice. A reestenose angiográfica binária foi definida pela presença de estenose em diâmetro maior que $50 \%$ ao reestudo angiográfico. A análise quantitativa das artérias coronárias foi realizada com o emprego do sistema CAAS-II (Pie Medical Imaging, Holanda), sendo efetuada no centro de pesquisa do Laboratório de Hemodinâmica do InCor.

\section{Análise com ultra-sonografia intravascular}

No momento do reestudo angiográfico, realizouse uma avaliação ultra-sonográfica intracoronária nos pacientes tratados com SEP. As imagens de ultra-sonografia intravascular foram obtidas com um cateter de ultra-som mecânico de $40 \mathrm{MHz}$ e 2,6 F de diâmetro (Atlantis $^{\mathrm{TM}}$, Boston Scientific Corp., Estados Unidos), conectado ao sistema de imagem de ultra-sonografia intravascular Galaxy II (Boston Scientific Corp., Estados Unidos), após a administração intracoronária de 0,1 mcg a 0,2 mcg de nitroglicerina, realizando a retirada com um sistema de pullback motorizado $(0,5 \mathrm{~mm} / \mathrm{s})$. As imagens foram gravadas continuamente ao longo do stent e incluíram, pelo menos, $5 \mathrm{~mm}$ das bordas proximal e distal ao stent. As imagens foram gravadas em sistema VHS e digitalizadas para o sistema DICOM, para realização de análises na estação de trabalho de ultrasonografia intravascular Curad IVUS Analysis Workstation Versão 3.29S (Pie Medical Imaging, Holanda). A análise foi executada no próprio centro executor do estudo. Medidas quantitativas foram obtidas no segmento do stent e nos segmentos de referência, incluindo: área da membrana elástica externa, área da luz, área seccional do stent e área de hiperplasia neointimal. A aposição incompleta do stent foi diagnosticada quando pelo menos uma haste do stent se encontrava separada da parede do vaso, com evidência de fluxo sanguíneo por trás do stent, na ausência de ramo lateral ${ }^{8}$.

\section{Análise estatística}

Variáveis categóricas foram apresentadas como valores absolutos e/ou porcentagens e comparadas com o teste exato de Fisher. Variáveis contínuas foram apresentadas como média \pm desvio padrão e comparadas 
com teste $t$ de Student. A sobrevida livre de ECAM foi estimada por meio do método de Kaplan-Meier e comparada com o teste de log-rank. O software estatístico empregado foi o SigmaStat (Systat Software Inc.). O nível de significância foi ajustado para $p=0,05$.

\section{RESULTADOS}

\section{Características basais}

As características basais e do procedimento foram bem balanceadas entre os grupos (Tabela 1). A média de idade foi de 59,0 $\pm 3,5$ anos e $76,7 \%$ dos pacientes eram homens. IAM com elevação do segmento ST foi a apresentação clínica principal (86,7\%). Um terço dos pacientes apresentava diabetes melito. O diâmetro médio dos stents foi de 3,07 $\pm 0,22 \mathrm{~mm}$ e o comprimento médio dos stents foi de 21,13 \pm 4,66 mm. Exame ecocardiográfico de rotina pós-IAM demonstrou fração de ejeção ventricular esquerda média de 69,2 \pm 14,0\%.

\section{Evolução clínica}

Não houve ECAM durante o período da internação hospitalar no procedimento índice. Os pacientes foram seguidos, prospectivamente, por um período de 24,9 $\pm 5,3$ meses. Houve uma morte não-cardíaca (câncer de pulmão), 27 meses após o procedimento índice, no grupo controle. A incidência de ECAM estimada ( $F i$ gura 2), aos 25 meses, foi de 3,3\% no grupo SEP e de $20,7 \%$ no grupo controle $(p=0,067)$. Onze meses após o procedimento índice, houve apenas um novo caso

TABELA 1

Características clínicas basais e do procedimento

\begin{tabular}{|c|c|c|c|}
\hline & Grupo SEP $(n=30)$ & Grupo controle $(n=30)$ & Valor de $p$ \\
\hline Idade (anos) & $58,8 \pm 10$ & $58,3 \pm 8,5$ & 0,9 \\
\hline Sexo masculino & 73,0 & 80,0 & 1,0 \\
\hline Diabetes & 33,3 & 33,3 & 1,0 \\
\hline Não-dependente de insulina & 23,3 & 26,7 & 0,8 \\
\hline Dependente de insulina & 10,0 & 6,7 & 1,0 \\
\hline Hipertensão & 86,7 & 80,0 & 0,7 \\
\hline Hipercolesterolemia & 76,6 & 70,0 & 0,7 \\
\hline Tabagismo & 63,3 & 50,0 & 0,4 \\
\hline Fração de ejeção do ventrículo esquerdo (\%) & $68,2 \pm 15,6$ & $70,4 \pm 11,9$ & 0,6 \\
\hline Quadro clínico & & & 1,0 \\
\hline IAM com supra de ST & 86,7 & 86,7 & \\
\hline IAM sem supra de ST & 13,3 & 13,3 & \\
\hline Tempo porta-balão (h) & $7,9 \pm 4,4$ & $8,6 \pm 5,0$ & 0,6 \\
\hline Padrão arterial (\%) & & & 0,8 \\
\hline Uniarterial & 16,7 & 23,3 & \\
\hline Biarterial & 76,7 & 70,0 & \\
\hline Triarterial & 6,7 & 6,7 & \\
\hline Vaso tratado $(\%)$ & & & 0,07 \\
\hline Coronária direita & 30,0 & 40,0 & \\
\hline Circunflexa & 6,7 & 23,3 & \\
\hline Descendente anterior & 63,3 & 36,7 & \\
\hline Fluxo TIMI pré-procedimento (\%) & & & 1,0 \\
\hline Grau $0-1$ & 40,0 & 36,7 & \\
\hline Grau 2 & 30,0 & 33,3 & \\
\hline Grau 3 & 30,0 & 30,0 & \\
\hline Fluxo TIMI pós-procedimento (\%) & & & 1,0 \\
\hline Grau 2 & 3,3 & 3,3 & \\
\hline Grau 3 & 96,7 & 96,7 & \\
\hline Diâmetro nominal do stent (mm) & $3,07 \pm 0,22$ & $3,08 \pm 0,23$ & 0,7 \\
\hline Comprimento do stent (mm) & $21,9 \pm 4,9$ & $20,4 \pm 4,4$ & 0,2 \\
\hline
\end{tabular}


Martino F de, et al. Segurança e Eficácia Tardia do Stent com Eluição de Paclitaxel no Infarto Agudo do Miocárdio: um Estudo CasoControle. Rev Bras Cardiol Invas 2007; 15(4): 343-349.

de IAM associado a reestenose do stent e lesão de tronco da artéria coronária esquerda no grupo controle, que foi tratado com revascularização cirúrgica de urgência. A revascularização da lesão-alvo foi necessária em 1 (3,3\%) paciente do grupo SEP e em 7 (23,3\%) pacientes do grupo controle $(p=0,02)$. Todas as reintervenções foram percutâneas, exceto por dois casos cirúrgicos no grupo controle. O paciente do grupo SEP que sofreu revascularização da lesão-alvo (nova angioplastia com stent convencional por reestenose da borda distal do stent) apresentou trombose tardia relacionada à porção distal do stent convencional implantado dois meses após o procedimento para revascularização da lesão-alvo, a despeito do uso de terapia antiplaquetária dupla. Esse paciente não apresentava aposição incompleta do stent ao estudo ultra-sonográfico.

\section{Evolução angiográfica}

O reestudo angiográfico foi realizado em todos os pacientes (Tabela 2). As características angiográficas quantitativas pré e pós-procedimento foram similares entre os grupos do estudo. Ao reestudo, o grupo SEP apresentou diâmetro luminal significativamente maior $(p=0,04)$, menor estenose em diâmetro $(p<0,01)$, menor perda tardia $(p=0,01)$ e menor índice de perda $(p<0,01)$ que o grupo controle. A taxa de reestenose binária foi de 3,3\% no grupo SEP e de 33,3\% no grupo controle $(p=0,002)$. Não foram identificados aneurismas de coronária em nenhum dos grupos.

\section{Avaliação com ultra-sonografia intracoronária}

O exame de ultra-sonografia intracoronária foi realizado em $23(76,7 \%)$ pacientes do grupo SEP. O volume do stent foi de $181,1 \pm 56,8 \mathrm{~mm}^{3}$ e o volume neointimal foi de $7,4 \pm 10,9 \mathrm{~mm}^{3}$. A porcentagem de obstrução neointimal intra-stent foi de 4,7 $\pm 6,8 \%$. A aposição incompleta tardia do stent (Figura 3) foi observada em $8(34,8 \%)$ pacientes e não se associou com ECAM.

\section{DISCUSSÃO}

O principal achado deste estudo, com seguimento angiográfico de todos os casos, foi que o implante de SEP para os pacientes com IAM foi seguro e efetivo em reduzir a ocorrência de reestenose tanto clínica como angiográfica, comparativamente a um grupo controle tratado com stent convencional análogo.

Os pacientes selecionados neste estudo não apresentavam instabilidade hemodinâmica e só foram incluídos após a passagem da corda-guia através da lesão de forma bem-sucedida. O perfil de risco relativamente baixo dos nossos pacientes resultou em taxa de mortalidade pequena durante o acompanhamento clínico. De fato, estudos prévios têm relatado mortalidade em seis meses extremamente pequena $(<1 \%)$ entre pacientes com IAM estratificados como baixo risco com base em suas características clínicas e angiográficas ${ }^{9}$. Entretanto, as taxas de reintervenção e de reestenose angiográfica

TABELA 2

Angiografia coronária quantitativa dos pacientes tratados com SEP ou stents convencionais

\begin{tabular}{|c|c|c|c|}
\hline & Grupo SEP $(n=30)$ & Grupo controle $(n=30)$ & Valor de $p$ \\
\hline \multicolumn{4}{|l|}{ Pré-procedimento } \\
\hline Diâmetro de referência (mm) & $2,67 \pm 0,42$ & $2,73 \pm 0,47$ & 0,6 \\
\hline Diâmetro luminal mínimo (mm) & $0,49 \pm 0,46$ & $0,49 \pm 0,46$ & 1,0 \\
\hline Estenose em diâmetro (\%) & $81,2 \pm 17,7$ & $81,6 \pm 16,9$ & 0,9 \\
\hline Comprimento da lesão (mm) & $17,2 \pm 5,9$ & $15,4 \pm 5,5$ & 0,3 \\
\hline \multicolumn{4}{|l|}{ Pós-procedimento } \\
\hline Diâmetro de referência (mm) & $2,81 \pm 0,43$ & $2,78 \pm 0,49$ & 0,8 \\
\hline Diâmetro luminal mínimo (mm) & $2,25 \pm 0,34$ & $2,28 \pm 0,41$ & 0,8 \\
\hline Estenose em diâmetro (\%) & $18,7 \pm 6,6$ & $18,8 \pm 6,7$ & 1,0 \\
\hline Ganho agudo (mm) & $1,77 \pm 0,58$ & $1,79 \pm 0,60$ & 0,9 \\
\hline \multicolumn{4}{|l|}{ Reestudo } \\
\hline Diâmetro de referência (mm) & $2,68 \pm 0,37$ & $2,83 \pm 0,48$ & 0,2 \\
\hline Diâmetro luminal mínimo (mm) & $2,04 \pm 0,38$ & $1,69 \pm 0,85$ & 0,04 \\
\hline Estenose em diâmetro (\%) & $24,1 \pm 9,90$ & $42,0 \pm 25,0$ & $<0,01$ \\
\hline Perda tardia (mm) & $0,21 \pm 0,29$ & $0,60 \pm 0,76$ & 0,01 \\
\hline Índice de perda (mm) & $0,11 \pm 0,14$ & $0,37 \pm 0,46$ & $<0,01$ \\
\hline Reestenose binária (\%) & 3,3 & 33,3 & $<0,01$ \\
\hline
\end{tabular}

Os números são expressos como média \pm desvio padrão ou como porcentagem.

SEP = stent com eluição de paclitaxel; $\mathrm{n}$ = número de pacientes. 


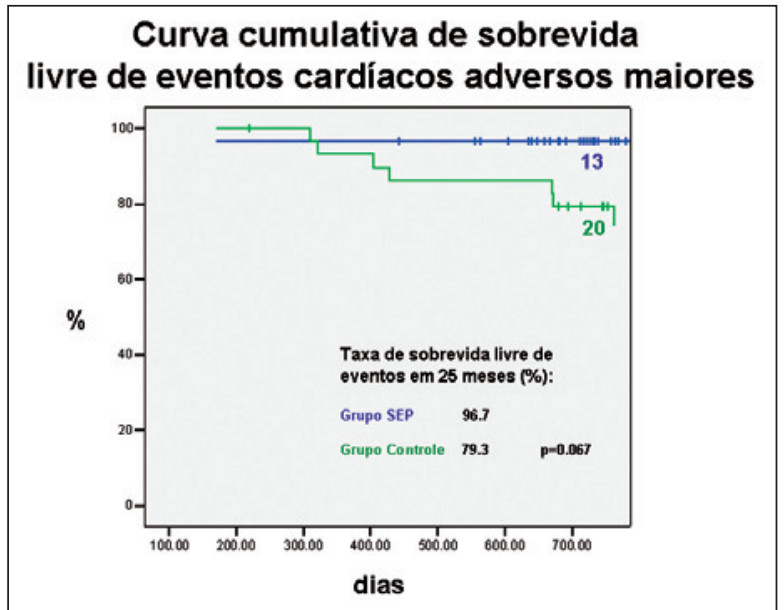

Figura 2 - Curva de Kaplan-Meier para análise de sobrevida livre de eventos cardíacos adversos maiores comparando o grupo SEP e o grupo controle.

\section{Aposição incompleta tardia do stent eluído com paclitaxel}

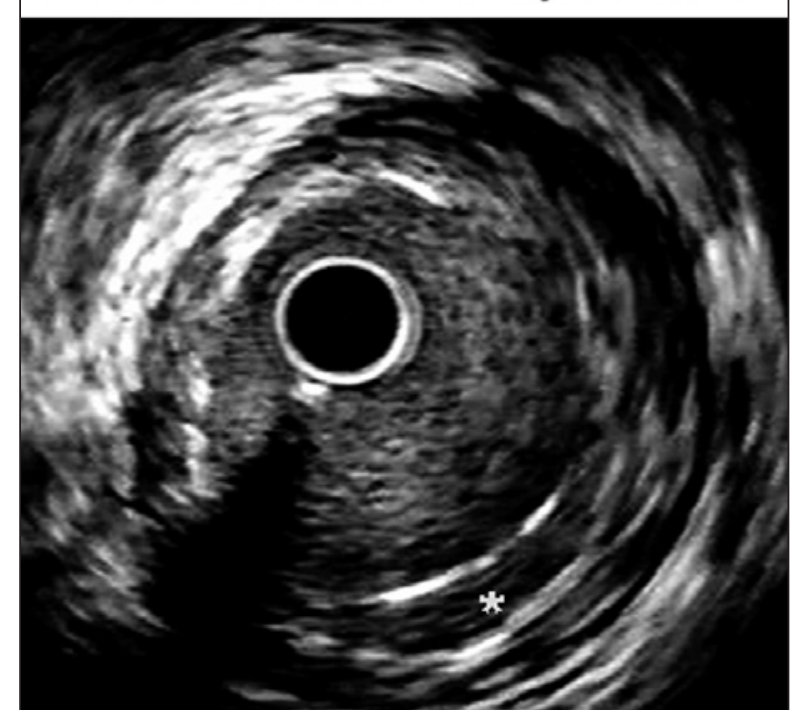

Figura 3 - Imagem de ultra-sonografia intracoronária da artéria descendente anterior obtida durante reestudo angiográfico tardio (oito meses após o procedimento índice) de paciente tratado com stent com eluição de paclitaxel (Taxus ${ }^{T M}$ ). O asterisco indica o local de aposição incompleta tardia do stent.

foram um pouco maiores que o esperado entre os pacientes do grupo controle (tratados com stents convencionais), o que pode refletir o reestudo angiográfico obrigatório, a elevada proporção de diabéticos, o tratamento de vasos de pequeno calibre e a ocorrência de lesões mais extensas que o relatado em séries prévias. Independentemente do exposto, o uso de SEP associou-se a baixa incidência de reestenose. De fato, a taxa de revascularização da lesão-alvo do grupo SEP (3,3\%) foi semelhante à relatada nos pacientes com síndrome coronária aguda do estudo TAXUS-IV (3,9\%), que não incluiu pacientes com IAM com supradesnivelamento do segmento $\mathrm{ST}^{3}$, e menor que a relatada no estudo PASSION $(5,3 \%)^{4}$.

Estudo ultra-sonográfico foi obtido em cerca de $75 \%$ dos pacientes do grupo SEP, em decorrência de dificuldades técnicas e logísticas inerentes à pesquisa clínica. Deve-se ressaltar, no entanto, que a análise ultrasonográfica não era uma meta primária do estudo. As análises de angiografia quantitativa e da ultra-sonografia intracoronária foram realizadas no próprio centro executor da pesquisa, o que também pode ser considerado uma limitação do estudo. Por outro lado, os resultados deste estudo não diferiram significativamente dos encontrados em estudos clássicos da literatura, que tiveram suas análises realizadas por centros independentes. Observou-se baixa taxa de reestenose binária $(3,3 \%)$ e pequena perda tardia $(0,21 \pm 0,29 \mathrm{~mm})$, bem como pequeno volume neointimal, à ultrasonografia intravascular no grupo SEP, semelhante ao que foi relatado em estudos anteriores com pacientes eletivos que receberam $\mathrm{SEP}^{1,2}$. Esse desempenho foi aparentemente alcançado sem incremento da taxa de trombose tardia do stent, durante seguimento clínico de dois anos. Resultados similares foram encontrados no estudo PASSION, após um ano de acompanhamento clínico ${ }^{4}$.

Estudos prévios com pacientes eletivos têm relatado taxas de má aposição tardia que variam entre $4 \%$ e $16 \%$ após implante de stents convencionais e de stents com eluição de sirolimo ou paclitaxel. Em pacientes com IAM, têm sido relatadas taxas três vezes maiores de má aposição tardia após implante de stent convencional, o que pode ser atribuído à reabsorção tardia do trombo no local de implantação do stent. Neste estudo, pacientes que receberam SEP para tratamento do IAM apresentaram incidência relativamente alta (aproximadamente 35\%) de má aposição tardia do stent ao exame ultra-sonográfico. Como a ultra-sonografia intracoronária encontrava-se disponível apenas para o grupo SEP, não foi possível realizar comparações entre os grupos SEP e controle. Por outro lado, à semelhança de estudos prévios, a má aposição tardia do stent não foi associada com qualquer evento durante o seguimento clínico.

O número reduzido de pacientes e a ausência de randomização são duas importantes limitações deste estudo. Embora o tamanho amostral relativamente pequeno possa ter limitado a capacidade de detecção de complicações raras relacionadas ao stent, o número de pacientes incluídos mostrou-se suficiente para identificar efeito estatisticamente significativo dos SEP para reduzir reestenose. O grupo controle selecionado foi bem pareado com relação às características clínicas e angiográficas pré e pós-procedimento. $\mathrm{O}$ desenho deste estudo permitiu uma análise razoável do desempenho 
dos SEP em pacientes com IAM de baixo risco e aumentou o conhecimento sobre a má aposição tardia dos SEP ${ }^{10}$.

\section{CONCLUSÕES}

O SEP (Taxus ${ }^{\mathrm{TM}}$ ) pareceu efetivo em reduzir a reestenose tanto clínica como angiográfica em pacientes com IAM, comparativamente aos stents convencionais. Embora a ocorrência de má aposição tardia do stent tenha sido relativamente elevada quando comparada a algumas séries históricas, isso não foi associado a aumento da incidência de ECAM durante o acompanhamento de dois anos. O implante de SEP parece ser uma estratégia segura e efetiva para reduzir a necessidade de novas intervenções em pacientes com IAM.

\section{REFERÊNCIAS BIBLIOGRÁFICAS}

1. Colombo A, Drzewiecki J, Banning A, Grube E, Hauptmann $\mathrm{K}$, Silber S, et al. Randomized study to assess the effectiveness of slow- and moderate-release polymer-based paclitaxeleluting stents for coronary artery lesions. Circulation. 2003; 108(7):788-94.

2. Stone GW, Ellis SG, Cox DA, Hermiller J, O'Shaughnessy C, Mann JT, et al. One-year clinical results with the slowrelease, polymer-based, paclitaxel-eluting TAXUS stent: the TAXUS-IV trial. Circulation. 2004;109(16):1942-7.

3. Moses JW, Mehran R, Nikolsky E, Lasala JM, Corey W, Albin $\mathrm{G}$, et al. Outcomes with the paclitaxel-eluting stent in patients with acute coronary syndromes: analysis from the TAXUS-IV trial. J Am Coll Cardiol. 2005;45(8):1165-71.

4. Laarman GJ, Suttorp MJ, Dirksen MT, van Heerebeek L,
Kiemeneij F, Slagboom T, et al. Paclitaxel-eluting versus uncoated stents in primary percutaneous coronary intervention. N Engl J Med. 2006;355(11):1105-13.

5. Hofma SH, Ong AT, Aoki J, van Mieghem CA, Rodriguez Granillo GA, Valgimigli $M$, et al. One year clinical follow up of paclitaxel eluting stents for acute myocardial infarction compared with sirolimus eluting stents. Heart. 2005; 91(9):1176-80.

6. Saia F, Lemos PA, Lee $\mathrm{CH}$, Arampatzis CA, Hoye A, Degertekin $M$, et al. Sirolimus-eluting stent implantation in ST-elevation acute myocardial infarction: a clinical and angiographic study. Circulation. 2003;108(16):1927-9.

7. Grines CL, Cox DA, Stone GW, Garcia E, Mattos LA, Giambartolomei A, et al. Coronary angioplasty with or without stent implantation for acute myocardial infarction. Stent Primary Angioplasty in Myocardial Infarction Study Group. N Engl J Med. 1999;341(26):1949-56.

8. Serruys PW, Degertekin M, Tanabe K, Abizaid A, Sousa JE, Colombo A, et al. Intravascular ultrasound findings in the multicenter, randomized, double-blind RAVEL (RAndomized study with the sirolimus-eluting VElocity balloon-expandable stent in the treatment of patients with de novo native coronary artery Lesions) trial. Circulation. 2002;106(7):798-803.

9. Grines CL, Marsalese DL, Brodie B, Griffin J, Donohoue B, Constantini CR, et al. Safety and cost-effectiveness of early discharge after primary angioplasty in low risk patients with acute myocardial infarction. PAMI-II Investigators. Primary Angioplasty in Myocardial Infarction. J Am Coll Cardiol. 1998;31(5):967-72.

10. Martino F, Ribeiro EE, Lemos PA, Perin MA, Padilha R, Prata S, et al. Paclitaxel-eluting stent implantation for acute myocardial infarction in comparison with conventional stenting: a clinical, angiographic, and IVUS prospective study. In: World Congress of Cardiology and European Society of Cardiology, 2006, Barcelona. Eur Heart J. 2006;27(S):650-1. 